\title{
Presencia de anti-VHE en un estudio de cohorte de porcinos ¿reservorio animal de hepatitis $\mathrm{E}$ en Chile?
}

\author{
H umberto Ibarra V, Stella Riedemann G a, German \\ Reinhardt Va, Mario Calvo A.
}

\section{Presence of anti hepatitis $E$ virus antibodies in swine}

Background: Swine hepatitis E virus (HEV) has a cross-reaction with human anti-HEV antibodies. Therefore, pigs could be an animal reservoir, rendering hepatitis $\mathrm{E}$ as a zoonosis. The spread of this infection among infected pigs across countries would be possible through trading. Previously, using an anti-human conjugate, we detected anti-HEV antobodies in adult pigs in Chile. Aim: To detect anti-HEV (ELSA) in a cohort of swine at different ages. Material and Methods: Two hundred pigs aged 42 to 360 days, divided in 20 groups of 10 animals were tested. Anti-HEV was detected by ELSA using anti-pig IgG horseradish peroxidase instead of anti-human conjugates. Results: Anti-HEV were detected in one animal aged 90 days, two animals aged 120 days, one animal aged 260 days and 2 animals aged 360 days, five pregnant sows and two old hogs. This represents a total of 14 animals or $7 \%$ of the sample. Conclusions: There is a significant prevalence of anti-HEV in pigs from 90 days of birth, suggesting that these swine are a probable reservoir (Rev Méd Chile 2007; 135: 997-1001).

(Key words: Antobodies, viral; Hepatitis E virus; Swine)

Recibido el 29 de agosto, 2005. Aceptado el 2 de marzo, 2007.

Trabajo financiado por Proyecto S-200379 DID-UACH.

Instituto de Medicina e Instituto de Microbiología, Universidad Austral de Chile. Valdivia, Chile. aédico Veterinario

L a hepatitis por virus E, de transmisión feco-oral, es un problema de salud pública en muchos países en desarrollo, ya que se asocia a condiciones sanitario ambientales deficientes. Sin embargo, en los últimos años han surgido problemas de infección de fuentes de alimentación derivados de enfermedades animales emergentes ${ }^{1}$ y la infección por virus hepatitis E (VHE) puede ser más fre-

Correspondencia a: Humberto Ibarra V. Casilla 567, Valdivia, Chile. Fax 63-221995. E mail hibarra@uach.cl cuente de lo considerado anteriormente en países industrializados ${ }^{2}$.

La mayor incidencia de hepatitis E ocure en adultos jóvenes, presentándose y evolucionando en forma similar a la hepatitis A. Pero la tasa de mortalidad en pacientes embarazadas es cercana a $20 \% 3,4$.

Por análisis filogenético, el VHE fue excluido de la familia Caliciviridae y los aislados virales del VHE se agrupan geográficamente en 4 genotipos: I (Myanmar, países de Asia, Africa), II (México, Nigenia); III (Estados Unidos de Norteamérica, Japón, Italia, Grecia, España, Argentina, Austria); IV (China, Taiwán) ${ }^{5-7}$. 
Experimentalmente se demostró que cerdos domésticos pueden ser infectados con aislados humanos del virus $\mathrm{E}^{8}$. En 1997, se aisló el VHE porcino (swine HEV), en cerdos de Estados Unidos de Norteamérica que reacciona en forma cruzada con anti-VHE humano ${ }^{9}$. El VHE porcino es considerado un nuevo agente zoonótico debido a su estrecha similitud genómica con el VHE humano y su capacidad de infectar primates no humanos ${ }^{7}$.

Se han identificado aislados de VHE porcino en cerdos de Japón, Nueva Zelandia, Corea, Holanda y Canadá, que junto al de Estados Unidos de Norteamérica se incluyen en el genotipo III y aislados de China y Taiwán incluidos en el genotipo IV ${ }^{10-15}$.

En médicos veterinarios que trabajan con porcinos, se ha encontrado prevalencia de anti-VHE 1,5 veces más alta que una población de donantes de sangre $^{16}$, por lo que son considerados como un grupo humano de riesgo, al igual que trabajadores en contacto con secreciones biológicas de animales y contacto con aguas servidas ${ }^{17-19}$.

Existen evidencias de anti-VHE en otras especies, como aves domésticas, roedores, gato ${ }^{20} \mathrm{y}$ recientemente se logró la identificación genética de un virus hepatitis en aves (avian HEV) ${ }^{21,22}$.

En Chile, la infección por VHE no es endémica y presenta una prevalencia de anti-VHE en donantes de sangre que varía entre $2 \%$ y $7 \% 23,24$. Respecto del estudio en cerdos en nuestro país, sólo existe una comunicación preliminar de prevalencia general, en que se detectó anti-VHE por ELISA, usando conjugado anti-humano ${ }^{25}$.

Estudios en América del Sur han detectado anti-VHE en cerdos para consumo humano, sugiriendo a esta especie como un reservorio para esta infección ${ }^{20,26}$.

El objetivo de esta investigación fue determinar anti-VHE en una cohorte de porcinos de un plantel de la zona central de Chile, de distintos grupos de edad, utilizando conjugado anti-porcino, y de esta manera aportar mayor información en este campo de investigación y evaluar su significado como reservorio en nuestro país.

\section{Material y MÉTodo}

El grupo de estudio, correspondió a 200 porcinos de un solo plantel de la zona central de Chile, con edades de entre 42 y 360 días y ejemplares hembras mayores de un año que se clasificaron por número de partos del $1^{\underline{0}}$ a $8^{0}$ parto. Los verracos viejos fueron ejemplares sobre 4 años. Todos estos animales se agruparon en 20 grupos de 10 ejemplares cada uno.

Se analizó en el suero la presencia de anti-VHE mediante ELISA de acuerdo las instrucciones del fabricante (Genelabs Diagnostics), pero se reemplazó un conjugado para ser aplicado en $\operatorname{cerdos}^{27}$. Brevemente, $10 \mu \mathrm{l}$ de muestra se agregaron a pocillo con $200 \mu$ l de diluyente, incubando la microplaca por $30 \mathrm{~min}$ a $37^{\circ} \mathrm{C}$. Posteriormente se lavó seis veces con $250 \mu \mathrm{l}$ de solución de lavado. A cada pocillo se agregó, en reemplazo del conjugado anti-humano, $100 \mu \mathrm{l}$ de conjugado antiporcino diluido al 1:1000 y se repitió procedimiento, agregándose después 100 ul de sustrato. Se incubó $15 \mathrm{~min}$ en oscuridad deteniendo la reacción con $50 \mu$ l de solución. Se efectuó lectura automática de absorbancia considerando positivo un valor sobre el cutoff.

\section{RESUlTADOS}

La aplicación de ELSA reemplazando conjugado anti-humano por conjugado anti-porcino detectó anti-VHE en un total de 14/200 (7\%). La positividad se pesquisó en 1 ejemplar de 90 días, 2 de 120 días, 1 de 260 y 2 de 360 días. Fue positivo también en hembras: 1 de $5^{\circ}$ parto, 2 de $6^{0}$ parto, 2 de $7^{0}$ parto y 1 de $8^{0}$ parto. Además fue positivo en 2 verracos viejos (Tabla 1 ).

\section{DisCUSIÓN}

En esta investigación, a diferencia de lo publicado anteriormente en Chile, utilizamos conjugado antiporcino, con lo cual detectamos anti-VHE en 7\% de estos ejemplares (14/200). La prevalencia se detectó en ejemplares a partir de los 90 días, edad en que los porcinos se comercializan para consumo humano, siendo estos resultados concordantes con lo previamente comunicado por otros autores $^{9}$. También observamos mayor positividad en porcinos a partir del 50 parto (Tabla 1). En un estudio anterior de porcinos de edad adulta, usando conjugado anti-humano, habíamos detec- 
Tabla 1. Resultados de anti-VH E en una cohorte de 200 porcinos

\begin{tabular}{|c|c|c|}
\hline Rangos de edad (días) & № porcinos & № anti-VHE + (\%) \\
\hline $42-49$ & 10 & $0(0 \%)$ \\
\hline $50-57$ & 10 & $0(0 \%)$ \\
\hline $58-65$ & 10 & $0(0 \%)$ \\
\hline $66-73$ & 10 & $0(0 \%)$ \\
\hline Días de edad & № porcinos & № anti-VHE + (\%) \\
\hline 90 & 10 & $1(10 \%)$ \\
\hline 120 & 10 & $2(20 \%)$ \\
\hline 150 & 10 & $0(0 \%)$ \\
\hline 210 & 10 & $0(0 \%)$ \\
\hline 260 & 10 & $1(10 \%)$ \\
\hline 360 & 10 & $2(20 \%)$ \\
\hline № partos & № porcinos & № anti-VHE + (\%) \\
\hline $1^{0}$ parto & 10 & $0(0 \%)$ \\
\hline 20 parto & 10 & $0(0 \%)$ \\
\hline 3o parto & 10 & $0(0 \%)$ \\
\hline 40 parto & 10 & $0(0 \%)$ \\
\hline 5o parto & 10 & $1(10 \%)$ \\
\hline 6o parto & 10 & $2(20 \%)$ \\
\hline 70 parto & 10 & $2(20 \%)$ \\
\hline $8^{\circ}$ parto & 10 & $1(10 \%)$ \\
\hline Verracos viejos & 10 & $2(20 \%)$ \\
\hline Total & 200 & $14(7 \%)$ \\
\hline
\end{tabular}

tado prevalencias entre $0,6 \%$ y $9,5 \%$ en 375 cerdos de distintas zonas del país ${ }^{25}$, con lo cual confirmamos que existe reacción cruzada al usar ambos métodos. Es decir, en Chile, con prevalencia más bien baja para VHE, poblaciones porcinas de consumo humano podrían ser un reservorio de VHE y eventual fuente de infección. En forma análoga, en países de alta endemicidad para VHE (anti-VHE 20\% en población humana aparentemente sana) se ha comunicado prevalencia de $72 \%$ en predios porcinos ${ }^{28}$.

La importancia de estos hallazgos debe analizarse considerando también que el VHE puede infectar cerdos precozmente y diseminar la infección a otros países a través de la importación de esta especie, tanto para su reproducción como para su consu$\mathrm{mo}^{29}$. En este sentido, en ciertas regiones de Estados
Unidos de Norteamérica se ha detectado alta prevalencia de anti-VHE en cerdos de uso comencial ${ }^{11}$. En América Latina, un estudio en 357 cerdos en el sur de Brasil, comunicó una prevalencia global de $63 \%$ y de $95 \%$ en cerdos de más de 20 semanas $^{20}$.

Experimentalmente se ha demostrado la transmisión por secreción feco-oral del VHE entre cerdos y no por otras secreciones animales ${ }^{30}$. Recientes investigaciones han revelado $99,7 \%$ de identidad de la secuencia genómica del VHE entre jabalíes y ciervos y previos aislados humanos que consumieron carne cruda de dichas especies en Japón, sugiriendo esta fuente de contagio para el hombre ${ }^{31}$.

Considerando adicionalmente que los cerdos se están utilizando experimentalmente para xenotransplante, se requiere evaluar regularmente si el VHE está presente en esta especie ${ }^{32}$. 
Tomando en cuenta los resultados de esta investigación, se necesitarín efectuar, por un lado, estudios prospectivos en cerdos en relación al momento de la seroconversión. Por otro lado, se requenina aplicar secuencias especiales para detectar el virus hepatitis E porcino mediante RT-PCR tanto en sangre como en heces de estos animales.

EL VHE puede diseminarse más de lo pensado previamente, existiendo una fuerte asociación entre aislados virales detectados en residuos líquidos de mataderos de cerdos y en pacientes que desarrollan hepatitis aguda ${ }^{33}$.

El consumo de leche no pasteurizada aparece también como factor de riesgo en personas con o sin exposición ocupacional a manipulación de cerdos ${ }^{34}$. Asimismo, utilizar aguas no tratadas sanitariamente para riego agrícola, ha sido demostrado como de especial riesgo para estos trabajadores ${ }^{18}$.

Un estudio preliminar efectuado en esta región, en un pequeño grupo humano de riesgo como son trabajadores de alcantarillado, detectó $11 \%$ de prevalencia de anti-VHE (Ibarra, comuni-

\section{REFERENCIAS}

1. Yamane I. Epidemics of emerging animal diseases and food-borne infection problem over the last 5 years in Japan. Ann NY Acad Sci 2006; 1081: 30-38.

2. Ciemente-Casares P, Pina S, Buti M, Jardi R, Martin M, BofU-MAS S ET AL. Hepatitis virus E epidemiology in industrialized countries. Emerg Infect Dis 2003; 9: 448-54.

3. Aggarwal R, Krawczynski K. Hepatitis E: an overview and recent advances in clinical and laboratory research. Gastroenterol hepatol 2000; 15: 9-20.

4. Hamid SS, Jafri SM, Khan H, ShaH H, Abbas Z, FIELDS H. Fulminant hepatic failure in pregnant woman. Acute fatty liver or acute viral hepatitis? J Hepatol 2000; 25: 20-7.

5. PringiE CR. Minutes of the $27^{\text {th }}$ Intemational Committee on Taxonomy of Viruses Meeting. Arch Virol 1998; 143: 1449-59.

6. BeRKE T, Matson DO. Reclassification of the Caliciviridae into distinct genera and exclusion of hepatitis $\mathrm{E}$ viruses from the family on the basis of comparative phylogenetic analysis. Arch Virol 2000; 145: 1421-36.

7. CHOI IS, Kwon HJ, SHIN NR, Yoo HS. Identification of Swine Hepatitis E Virus (HEV) and Prevalence of Anti-HEV Antibodies in Swine and Human cación personal). Este porcentaje concordaría con estudios efectuados en otros países, en los cuales la positividad para anti-VHE, se ha correlacionado con el tipo de trabajo como factor independiente, también con el número de años de trabajo, pero no claramente asociado a la edad17,18,23. Se requieren más estudios epidemiológicos en nuestro país, que den información sobre más grupos de riesgo como veterinarios o trabajadores en contacto con secreciones biológicas de animales.

En Argentina ${ }^{26}$ han identificado el primer VHE porcino de Sudamérica en un predio de la provincia de Buenos Aires, sugiriendo que el cerdo puede ser un reservorio animal para esta infección. Este aislado perteneciente al genotipo III, se relaciona estrechamente con aislado de VHE de Austria, lo cual podría significar un origen europeo de esta infección.

De acuerdo a todas las evidencias anteriores, en Chile pudiera darse una situación epidemiológica similar a nuestros países vecinos, lo cual debiera estimular la investigación del VHE tanto en seres humanos como en especies animales.

Populations in Korea. J Clin Microbiol 2003; 41: 3602-8.

8. Balayan MS, Usmanov RK, Zamyatina DI, Karas FR. Brief report: experimental hepatitis $\mathrm{E}$ infection in domestic pigs. J Med Virol 1990; 32: 58-9.

9. Meng XJ, Purcell RH, Haibur PG, Lfhman JR, Webb DM, Tsareva TS ET AL. A novel virus in swine is closely related to the human hepatitis E virus. Proc Natl Acad Sci USA 1997; 94: 9860-65.

10. Окамото $\mathrm{H}$, Takahasi $\mathrm{M}$, Nishikawa $\mathrm{T}$, FuKai $\mathrm{K}$, Muramatsu U, YoshiKawa A. Analysis of the complete genome of indigenous swine hepatitis $\mathrm{E}$ virus isolated in Japan. Biochem Biophys Res Commun 2001; 289: 929-36.

11. Huang FF, Hagshenas G, Guenette DK, Haibur PG, Schommer SK, Person FW et al. Detection by reverse transcription-PCR and genetic characterization of fields isolates of swine hepatitis $\mathrm{E}$ virus from pigs in different geographic regions of the United States. J Clin Microbiol 2002; 40: 1326-32.

12. Van Der Poel WHM, Verschoor F, Vander Heide R, HerRera MI, Vivo A, Kooreman Met al. Hepatitis E virus sequences in swine related to sequences in humans, the Netherlands. Emerg Infect Dis 2001; 7: 970-6.

13. Garkavenko O, Obriadina A, Meng J, Anderson DA, Benard HJ, Schroeder BA et al. Detection and 
characterization of swine hepatitis E virus in New Zealand. J Med Virol 2001; 65: 525-9.

14. Hsieh SY, Meng XJ, Wu YH, Liu ST, TAm AW, LiN DY ET AL. Identity of a novel swine hepatitis E virus in Taiwan forming a monophyletic group with Taiwan Isolates of human hepatitis E virus. J Clin Microbiol 1999; 37: 3828-34.

15. PeI Y, Yoo D. Genetic characterization and sequence heterogeneity of a Canadian isolate of Swine hepatitis E virus. J Clin Microbiol 2002; 40: 4021-9.

16. Meng XJ, Wiseman B, Elvinger F, Guenette DK, Toth TE, Engle RE et al. Prevalence of Antibodies to Hepatitis E Virus in Veterinarians Working with Swine and in Normal Blood Donors in the United States and Other Countries. J Clin Microbiol 2002; 40: $117-22$.

17. Viddósola H, Colchon A, Barreda M, Piscoya J, Palacios O. Hepatitis E IgG antibodies seroprevalence in peruvian risk group. Rev Gastroenterol Peru 2000; 20: 111-16.

18. Ceylan A, Ertem M, Ilcin E, Ozekinci T. A special risk group for hepatitis E infection: Turkish agriculture workers who use untreated waste water for irrigation. Epidemiol Infect 2003; 131: 753-6.

19. Vaidya SR, Tilekar BN, Walimbe AM, Arankalie VA. Increased risk of hepatitis $E$ in sewage workers from India. J Occup Environ Med 2003; 45; 1167-70.

20. Vitral CL, Pinto MA, Lewis-Ximenez LL, Khudyakov YE, Dos SAntos DR, Gaspar AM. Serological evidence of hepatitis $E$ virus infection in different animal species from the Southeast of Brazil. Mem Inst Oswaldo Cruz 2005; 100: 117-22.

21. Huang FF, Sun ZF, Emerson SU, Purcell RH, ShivaPRASAD HL, Pierson FW ET al. Determination and analysis of the complete genomic sequence of avian hepatitis E virus (avian HEV) and attempts to infect rhesus monkeys with avian HEV. J Gen Virol 2004; 85: 1609-18.

22. Sun ZF, Larsen CT, Dunlop A, Huang FF, Pierson FW, Tотн TE ET AL. Genetic identification of avian hepatitis E virus (HEV) from healthy chicken flocks and characterization of the capsid gene of 14 avian HEV isolates from chickens with hepatitis-splenomegaly syndrome in different geographical regions of the United States. J Gen Virol 2004; 85: 693-700.

23. IbarRa $H$, Riedemann $S$, Reinhardt G, Frick P, Siegel F, Tolfedo C et al. Prevalencia de anticuerpos del virus Hepatitis $\mathrm{E}$ en donantes de bancos de sangre y otros grupos de población, en la X región, Chile. Rev Méd Chile 1997; 125: 275-8.

24. Brahm J, Hurtado C, Moraga M, Gil LC, Veiasco M, Aifgría S ET aL. Infección con el virus de la hepatitis E en Chile. Rev Méd Chile 1996; 124: 947-9.

25. Reinhardt G, Ibarra H, Ruedemann S, Vega I. Estudio serológico preliminar de hepatitis $\mathrm{E}$ en cerdos en Chile. Arch Med Vet 2003; 2: 233-6.

26. Munne MS, Vladimirsky S, Otegui L, Castro R, Brajterman L, Soto $\mathrm{S}$ ET al. Identification of the first strain of swine hepatitis E virus in South America and prevalence of anti-HEV antibodies in swine in Argentina. J Med Virol 2006; 78: 1579-83.

27. Ciayson ET, Innis BL, Myint KSA, Narupiti S, Vaughn DW, GIRI S ET AL. Detection of hepatitis E virus infections among domestic swine in the Kathmandu Valley of Nepal. Am J Trop Med Hyg 1995; 53: 228-32.

28. Wibawa ID, Muljono DH, Mulyanto, Suryadarma IG, Tsuda F, Takahasi M et al. Prevalence of antibodies to hepatitis E virus among apparently healthy humans and pigs in Bali, Indonesia: Identification of a pig infected with a genotype 4 hepatitis E virus. J Med Virol 2004; 73: 38-44.

29. Wu JC, Chen CM, Chiang TY, Tsai WH, Jeng WJ, SHEEN IJ ET AL. Spread of hepatitis E virus among different-aged pigs: two-year survey in Taiwan. J Med Virol 2002; 66: 488-92.

30. Kasorndorkbua C, Guenette DK, Huang FF, Thomas PJ, Meng XJ, Halbur PG. Routes of transmission of swine hepatitis E virus in pigs. J Clin Microbiol 2004; 42: 5047-52.

31. Takahashi K, Kitajima N, Abe N, Mishiro S. Complete or near complete nucleotide sequences of hepatitis $\mathrm{E}$ virus genome recovered from a wild boar, a deer, and tour patients who ate the deer. Virology 2004; 330: 501-5.

32. Tanaka $H$, Yoshino $H$, Kobayashi E, Takahashi $M$, Окамото H. Molecular investigation of hepatitis $\mathrm{E}$ virus infection in domestic and miniature pigs used for medical experiments. Xenotransplantation 2004; 11: 503-10.

33. Pina S, Buti M, Cotrina M, Pielia J, Gitones R. HEV identified in serum from humans with acute hepatitis and sewage of animal origin in Spain. J Hepatol 2000; 33: 826-33.

34. Drobeniuc J, Favorov Mo, Shapiro CN, Bell BP, MAST EE, DADU A ET AL. Hepatitis E virus antibody prevalence among persons who work with swine. J Infect Dis 2001; 184: 1594-7. 